\section{RMD Open}

Rheumatic \&

Musculoskeletal Diseases

\title{
Therapeutic and diagnostic outcomes of a standardised, comprehensive care pathway for patients with systemic sclerosis
}

\author{
Jessica Meijs, ${ }^{1}$ Anne A Schouffoer, ${ }^{1,2}$ Nina Ajmone Marsan, ${ }^{3}$ Lucia J M Kroft, ${ }^{4}$ \\ Theo Stijnen, ${ }^{5}$ Maarten K Ninaber, ${ }^{6}$ Tom W J Huizinga, ${ }^{1}$ \\ Theodora P M Vliet Vlieland, ${ }^{7}$ Jeska $\mathrm{K}$ de Vries-Bouwstra ${ }^{1}$
}

To cite: Meijs J, Schouffoer AA, Ajmone Marsan N, et al. Therapeutic and diagnostic outcomes of a standardised, comprehensive care pathway for patients with systemic sclerosis. RMD Open 2016;2:e000159. doi:10.1136/rmdopen-2015000159

- Prepublication history and additional material is available. To view please visit the journal (http://dx.doi.org/ 10.1136/rmdopen-2015000159).

Received 29 July 2015 Revised 17 February 2016 Accepted 20 February 2016

CrossMark

For numbered affiliations see end of article.

\section{Correspondence to} Dr Jeska K de VriesBouwstra; j.k.de_vriesbouwstra@lumc.nl

\section{ABSTRACT}

Objectives: To determine the outcomes, including number of medical interventions and initiation of immunosuppressive treatment of a standardised, comprehensive, diagnostic care pathway for patients with systemic sclerosis (SSc). Patient characteristics associated with need for medical interventions and with need for immunosuppressive treatment were determined. Methods: Data were routinely gathered in connection with a 2-day care pathway combining multidisciplinary care and complete diagnostic work-up of organ involvement in SSc. The number of patients in whom the pathway resulted in medical interventions, and/or initiation of immunosuppressives was recorded. Patient characteristics and diagnostic tests results were compared between patients with and without medical interventions, and patients with and without initiation of immunosuppressives by means of multivariable logistic regression analyses.

Results: During a period of 44 months, 226 patients with SSc were referred to the care pathway. They included $186(82 \%)$ women with mean age of 54 (SD 14.5) years, and median disease duration of 4 years (range 1-11); 73 (32\%) of them had diffuse cutaneous SSc. Medical interventions were initiated in 191 (85\%) patients, including initiation of immunosuppressive treatment in $n=49(22 \%)$. Presence of telangiectasias and higher erythrocyte sedimentation rate were associated with any medical intervention. Of commonly available variables, lower age, higher skin score and absence of anticentromere antibody were associated with initiation of immunosuppressives.

Conclusions: A standardised comprehensive 2-day care pathway for patients with SSc resulted in additional diagnostic or therapeutic interventions in $85 \%$ of the patients, regardless of SSc subtype and disease duration. In $22 \%$ of the patients, immunosuppressive treatment was initiated.

\section{INTRODUCTION}

Systemic sclerosis $(\mathrm{SSc})$ is a rare, multisystem connective tissue disorder which is highly

\section{Key messages}

- Standardised and regular screening for organ involvement is advocated in systemic sclerosis (SSc), and contributes to prolonged survival.

- Patients with SSc prefer regular standardised diagnostic testing in a day-care setting.

- An annual, comprehensive diagnostic and multidisciplinary 2-day care pathway was developed for patients with SSc.

- First evaluation in this care pathway resulted in additional diagnostic or therapeutic interventions in $85 \%$ of the patients.

- Initiation of interventions was independent of SSc subtype and disease duration, indicating usefulness of comprehensive care for all patients with SSc.

heterogeneous in presentation and severity, ranging from limited forms to diffuse disease involving vital visceral organs. ${ }^{1}{ }^{2}$ The frequency and diverse nature of the organ-based complications make systematic assessment and long-term follow-up essential for good management of SSc.

Recent studies have demonstrated an improvement in the overall survival among patients with SSc over the last few decades. ${ }^{34}$ Apart from improved survival of the population in general, ${ }^{1}$ different other factors, including greater awareness for organ involvement and better management of complications are thought to contribute to this improvement. ${ }^{4}$ As mortality in SSc from 5-year follow-up onwards is mainly determined by presence of organ complications, ${ }^{4}$ regular follow-up of patients with SSc, including screening for organ involvement seems justified. Indeed, current guidelines advocate a screening, including assessment of organ involvement in patients with SSc, to be 
performed at least annually. ${ }^{5-8}$ As risk for different organ-based complications varies between subsets of SSc the most extensive annual screening is advocated for patients at high risk for organ involvement. For these patients, and for patients newly presenting with SSc, annual screening, including evaluation of pulmonary, renal, cardiac, gastrointestinal and vascular complications is generally advocated. ${ }^{79-11}$

In addition, a range of SSc-specific manifestations, including, for example, Raynaud's phenomenon (RP) atrophy, joint contractures and digital ulcers, result in specific limitations in everyday life, for which additional counselling and non-pharmacological interventions can be relevant. Although evidence for efficacy of nonpharmacological interventions is limited, ${ }^{12}$ potential importance of psychosocial and rehabilitation interventions for a chronic disease are clear. ${ }^{13}$

To improve healthcare for patients with SSc treated in the Leiden University Medical Center (LUMC), we aimed to develop a care pathway combining comprehensive medical assessment of organ complications with multidisciplinary care specifically targeting SSc-related manifestations.

The execution of a care pathway combining a wide range of diagnostic procedures with multidisciplinary team care in a patient-friendly manner is an organisational challenge. Patients were found to prefer a yearly standardised, multidisciplinary assessment in a day-care setting rather than multiple visits to various outpatient clinics. ${ }^{14}$ In line with this preference, a yearly, 2-day standardised diagnostic and multidisciplinary care pathway was developed. It is meant to serve both patients from the university hospital as well as those referred by rheumatologists in non-academic hospitals in the region. The execution of this complex diagnostic and multidisciplinary care pathway in one centre is in line with international management guidelines, which advocate referral of patients with SSc to a specialised centre. $^{5}$

Regarding the therapeutic management resulting from the diagnostic care pathway, the 2009 EULAR guidelines on treatment of SSc, listing evidence and consensus-based pharmacological treatments for various manifestations of SSc, constitute a firm base. ${ }^{15}$ However, no clear definitions on required severity of manifestations of SSc with regard to start of treatments are available, which is due to the heterogeneity and complexity of the disease. In the absence of such definitions, all management decisions in the context of the above mentioned care pathway were taken at a multidisciplinary team conference, based on the individual patient characteristics, and all available scientific and clinical knowledge among the team members.

The aim of the current report is to determine the outcomes of this standardised, comprehensive, care pathway in terms of medical and paramedical interventions, and initiation of immunosuppressive treatment.
MATERIALS AND METHODS

Study design

Data for the present study were prospectively and routinely gathered as part of the standardised, annual 2-day care pathway. The present analysis only concerned the short-term outcomes of patients taking part in the care pathway for the first time between April 2009 and December 2012. Ethical approval was obtained from the Institutional Review Board of the LUMC. All participants gave written informed consent.

\section{Patients}

Admission criteria for the care pathway include a diagnosis of SSc according to the referring rheumatologist, or a strong suspicion for SSc, and a request for a complete diagnostic work-up to confirm the diagnosis. Patients can be referred by rheumatologists from the outpatient clinic of the LUMC or from any other hospital in the Netherlands.

\section{The 2-day care pathway}

The care pathway was started in 2009 (and is still operational now, in 2016). It comprises a visit to the rheumatologist, pulmonologist and cardiologist. In addition to the extensive medical screening, patients are routinely seen by a physical therapist, and a specialised nurse. Additionally, consultations with a social worker and/or occupational therapist are scheduled by indication. The specialised nurse acts as the personal manager and coordinator for the patients during the 2-day programme, explores specific information and healthcare needs, and provides individual counselling and instructions.

For every patient, the healthcare pathway is performed on 2 consecutive days between 8:00 and approximately 16:00. Patients not living in the area of Leiden are offered the opportunity to stay overnight in a hotel room inside the hospital. At initiation of the care pathway, the maximum capacity was 2 patients per week; based on the number of referrals, the capacity was increased to 4 patients weekly in 2012. Two weeks after completion of the care pathway, findings for each particular patient are thoroughly evaluated during a multidisciplinary team conference, attended by at least two rheumatologists, one pulmonologist and one specialised nurse, who is also informed about findings of the occupational therapist, the physical therapist, and the social worker.

\section{Assessments}

All assessments are performed as part of routine care. They include questionnaires to be completed by patients, as well as interviews and physical performance tests, laboratory tests, nailfold videocapillaroscopy (NVC) and imaging. 


\section{Sociodemographic characteristics}

For all patients, the following social and demographic characteristics are recorded: age, origin, smoking habit, use of alcohol and caffeine and educational level ((1) primary education (0-8 years; low education level), (2) secondary education (9-16 years; medium education level), or (3) higher vocational education/university (postsecondary; high education level)).

\section{SSc classification and severity}

Patients included for the current analysis all fulfil diagnostic criteria for SSc as defined by the American College of Rheumatology ${ }^{16}$ or LeRoy and Medsger ${ }^{17}$ criteria for (early) SSc. Duration of RP, duration of non-RP (time since first symptom other than RP), ${ }^{18}$ disease duration (time since diagnosis SSc was confirmed by a physician), physical complaints and modified Rodnan Skin Score (mRSS), are determined by an experienced rheumatologist.

NVC is routinely performed in all patients starting from 2011 with qualitative assessment of capillary patterns: normal/aspecific pattern, SSc pattern (early/ active/late) or borderline changes. ${ }^{19}$

\section{Physical functioning}

Patients are asked to fill in the Scleroderma Health Assessment Questionnaire; a 20-item questionnaire comprising eight domains of activities of daily living, with the final score ranging from 0 (no disability) to 3 (severe disability). ${ }^{20}$

In addition, the 6 min walk distance (6MWD) is determined. The 6MWD evaluates the global and integrated responses of all the systems involved during exercise, including pulmonary and cardiovascular systems, systemic and peripheral circulation, blood, neuromuscular units and muscle metabolism. ${ }^{21}$ It reflects daily exercise, and has good construct validity, as demonstrated in patients with SSc with pulmonary arterial hypertension $(\mathrm{PAH})$.

\section{Quality of life}

Quality of life is measured with the SF-36, which contains eight subscales. ${ }^{22}$ The scoring range of the SF-36 subscales is $(0-100)$, with higher scores indicating better quality of life. The subscales are converted into two summary scales: the physical component summary, and the mental component summary scale, standardised to a score with a mean of 50 and a SD of 10 in the general population. For that purpose, the scores from an agematched and sex-matched, normative sample, drawn from a large, random, nationwide sample of adults $(n=1742)$ from the general Dutch population frequency table and factor score coefficients are used. ${ }^{23}$

\section{Laboratory investigations}

- Measurement of haemoglobin, erythrocyte sedimentation rate (ESR), $\mathrm{C}$ reactive protein (CRP), creatine phosphokinase (CPK), NT-pro brain natriuretic peptide, creatinine and estimated glomerular filtration rate.

- Measurement of autoantibodies, including antinuclear antibody (indirect immunofluorescence on HEP-2000 cells (Biomedical Diagnostics, Antwerpen, Belgium)), antitopoisomerase I (anti-Scl-70) and anticentromere (ACA), both measured with Enzyme-Linked Immuno Assay technique (Immunocap 250, ThermoFisher Scientific, Nieuwegein, The Netherlands). Anti-RNA polymerase III was measured externally (ELISA; Sanquin, Amsterdam, The Netherlands); urine screening for erythrocyturia and proteinuria; $24 \mathrm{~h}$ urine collection.

\section{Cardiopulmonary investigations}

- High-resolution CT (HRCT) of the thorax, pulmonary function tests (PFTs; including analyses of vital capacity (VC), and diffusing capacity for carbon monoxide (DLCO)), cardiopulmonary exercise test (CPET; including analyses of maximum heart rate, maximum wattage, maximum oxygen consumption $\left(\mathrm{VO}_{2}\right)$ and maximum ventilation),

- Echocardiography, ECG and $24 \mathrm{~h}$ rhythm (Holter) registration.

Diagnosis of interstitial lung disease (ILD) is determined based on the presence of a non-specific interstitial pneumonia (NSIP) pattern, or usual interstitial pneumonia (UIP) pattern on HRCT thorax ${ }^{24}$ as reported by the radiologist.

The systolic pulmonary artery pressure (SPAP) is estimated using echocardiography by an experienced cardiologist, and elevated pulmonary pressure is defined using a cut-off value of $35 \mathrm{~mm} \mathrm{Hg} .{ }^{25}$ Left ventricular end-diastolic and end-systolic volumes are measured using the biplane modified Simpson's rule. Left ventricular ejection fraction is calculated as left ventricular end-diastolic - LV end-systolic volume/left ventricular end-diastolic volume. Furthermore, the presence of pericardial effusion is noted.

Presence of arrhythmias is defined as presence of multiform ventricular extrasystoles $>100 /$ day, couplets or runs of ventricular tachycardia or supraventricular tachycardia of at least $30 \mathrm{~s}$ on $24 \mathrm{~h}$ Holter monitoring. Conduction abnormalities are defined as complete left bundle branch block or right bundle branch block, atrioventricular block (first second or third degree), or pacemaker rhythm for sinus node dysfunction. ${ }^{26}$

\section{Diagnostic and/or therapeutic management strategies resulting from the care pathway}

The multidisciplinary team conference results in specific advices on (1) diagnostic follow-up, (2) referral to medical specialists, (3) referral to other healthcare professionals or (4) initiation of treatment, or immunosuppressive treatment, and change of supportive medication, including treatment for peripheral vascular complications, for each individual patient. Advice on diagnostic follow-up comprises all patients in whom 
additional diagnostic procedures are advocated during the next 12 months, including right heart catheterisation for possible PAH, gastroscopy, HRCT thorax or PFT for follow-up on pulmonary involvement. Patients with suspicion for possible PAH based on echocardiography, are additionally discussed in the multidisciplinary PAH meeting attended by the rheumatologist, cardiologist, pulmonologist and internist in order to discuss the indication for a right heart catheterisation (RHC).

Referral to medical specialists includes all patients in whom follow-up and treatment by medical specialists additional to follow-up by a rheumatologist is advocated. The decision to initiate immunosuppressive medication is based on consensus between experts during the multidisciplinary team conference in absence of a gold standard precisely guiding treatment decisions in SSc. Initiation of new immunosuppressive treatment is mainly considered in case of extensive and/or progressive skin or organ involvement, for example, in case of relevant decline in VC and/or DLCO (without using an absolute threshold) in combination with the presence of NSIP and/or UIP on HRCT. Autologous haematopoietic stem cell transplantation (HSCT) is applied according to inclusion criteria and treatment regimen, as described in the ASTIS trial. ${ }^{27}$ Azathioprine (AZA) is prescribed in case of active arthritis or pulmonary involvement, with contraindication for cyclophosphamide or mycophenolate mofetil; hydroxychloroquine (HCQ) is prescribed in case of SSc overlap syndrome with rheumatoid arthritis (RA). Rituximab is given either in patients with SSc overlap syndrome with RA, or as part of a randomised placebo-controlled clinical trial (RITIS), registered at http://www.clinicaltrialsregister.eu/ EudraCT Number: 2008-007180-16. Treatment for peripheral vascular complications included: bosentan, sildenafil, intravenous iloprost, calcium channel blockers and antibiotics (the latter in case of active digital ulcers with superimposed bacterial infection).

On the basis of the individual patient's need, the specialised nurse discussed specific non-pharmacological treatment advices regarding smoking habits, Raynaud's phenomenon (including prescription of therapeutic gloves), care for digital ulcers, and dietary advices.

In addition, patients with specific problems regarding activities or work participation, and/or psychosocial problems, which could not be solved by a single consultation or advice during the care pathway, were referred to the appropriate health professionals, according to the nature and severity of their problems, as well as their personal preferences.

\section{Patient satisfaction}

As part of quality management of the day care department, patients who participated in the care pathway were requested to complete a questionnaire concerning different aspects of the care pathway and the healthcare providers involved (see online supplementary file). All patients received this questionnaire on the second day of the care pathway, including a return envelope. For the current analyses, questions concerning patients' satisfaction with the care pathway and healthcare providers as such were evaluated.

\section{Statistical analysis}

According to their distribution, continuous variables are either presented as mean and SD or medians with IQR (p25-75). Categorical variables are presented as frequencies with percentages.

To determine which patients benefit most from the care pathway in terms of specific medical interventions (defined as: diagnostic follow-up, referral or change of treatment) and initiation of immunosuppressive treatment following the care pathway, patients with and without medical intervention, and patients with and without initiation of immunosuppressives were compared. Univariable and multivariable regression analyses were used to determine characteristics associated with the initiation of any medical intervention and with the initiation of immunosuppressive medication. To identify patient characteristics commonly available from history taking, physical examination and laboratory testing, and thus help to identify those patients who benefit most from the care pathway, multivariate regression analyses were also performed excluding results generated by diagnostic tests performed as part of the care programme. Comparisons between the characteristics of patients who did and who did not return the satisfaction questionnaire were done by means of unpaired t tests, Mann-Whitney U tests, or $\chi^{2}$ tests, where appropriate. For all data, an available case analysis was performed. Data entry was performed using Microsoft Office Access 2003. All statistical analyses were executed using SPSS V.20.0 software (SPSS Inc, Chicago, USA).

\section{RESULTS}

\section{Patient population}

Two hundred and forty patients were referred to the healthcare programme between 2009 and 2012. The diagnosis SSc was confirmed in 226 patients. These patients were mostly women $(\mathrm{n}=186,82 \%)$, Caucasian $(\mathrm{n}=151,67 \%)$ and, on average, 54 years old (SD 14.5). Patients had a median disease duration of 4 years (range $1-11$ years) and 73 of them (32\%) had DcSSc (table 1).

\section{Adherence with the care pathway}

All patients filled in the questionnaires and underwent laboratory investigations, ECG, 6MWD, HRCT, PFT and echocardiography.

Evaluation by CPET was performed in 209 patients (92\%), since 17 patients were not able to cycle, mainly caused by musculoskeletal disability. NVC was performed in 86 patients $(38 \%)$, of whom 78 patients $(91 \%)$ had a SSc pattern. An early pattern was seen in 10 patients, an active pattern in 37 patients and a late pattern in 31 patients. 
Table 1 Baseline characteristics of patients with systemic sclerosis

\begin{tabular}{|c|c|c|c|}
\hline & $\begin{array}{l}\text { Total } \\
\mathrm{N}=226\end{array}$ & $\begin{array}{l}\text { Medical } \\
\text { intervention } \\
\mathrm{N}=191\end{array}$ & $\begin{array}{l}\text { No medical } \\
\text { intervention } \\
\mathrm{N}=35\end{array}$ \\
\hline \multicolumn{4}{|l|}{ Sociodemographics } \\
\hline Age, years, mean (SD) & $54(14.5)$ & $54.5(14.9)$ & $51.4(11.5)$ \\
\hline Female, N (\%) & $186(82)$ & $157(82)$ & $29(83)$ \\
\hline Caucasian origin, $\mathrm{N}(\%)$ & $151(67)$ & $127(67)$ & $24(69)$ \\
\hline Smoking, current, N (\%) & $32(14)$ & $28(15)$ & $4(11)$ \\
\hline \multicolumn{4}{|l|}{ Education level, $\mathrm{N}(\%)^{\star}$} \\
\hline Low & $89(41)$ & $76(41)$ & $13(39)$ \\
\hline Medium & $78(36)$ & $68(36)$ & $10(30)$ \\
\hline High & $53(24)$ & $43(23)$ & $10(30)$ \\
\hline \multicolumn{4}{|l|}{ Disease characteristics, N (\%) } \\
\hline DcSSc & $73(32)$ & $62(33)$ & $11(31)$ \\
\hline mRSS, median (IQR) & $3(1-6)$ & $4(1-6)$ & $2(2-6)$ \\
\hline Disease duration, years, median (IQR) & $4(1-11)$ & $3(1-11)$ & $4(1-10)$ \\
\hline Onset of Raynaud's phenomenon, years, median (IQR)* & $12(5-20)$ & $12(5-20)$ & $10(5-201)$ \\
\hline $\begin{array}{l}\text { Onset of non-Raynaud's phenomenon, years, median } \\
\text { (IQR)† }\end{array}$ & $6(3-13)$ & $6(2-13)$ & $6(3-11)$ \\
\hline Digital ulcers & $62(28)$ & $57(30)$ & $5(14)$ \\
\hline Telangiectasia & $133(59)$ & $118(62)$ & $15(43)$ \\
\hline Synovitis & $23(10)$ & $21(11)$ & $2(6)$ \\
\hline Tendon friction rubs & $8(4)$ & $6(3)$ & $2(6)$ \\
\hline Proximal muscular weakness & $14(6)$ & $13(7)$ & $1(3)$ \\
\hline Pulmonary crackles & $64(29)$ & $57(30)$ & $7(20)$ \\
\hline Renal crisis & $10(5)$ & $10(5)$ & $0(0)$ \\
\hline \multicolumn{4}{|l|}{ Autoantibodies, N (\%) } \\
\hline ANA positivity & $209(93)$ & $177(93)$ & $32(91)$ \\
\hline Anti-Scl-70 positivity $\ddagger$ & $50(26)$ & $43(27)$ & $7(21)$ \\
\hline Anti-centromere positivity $\ddagger$ & 77 (40) & $62(38)$ & $15(48)$ \\
\hline \multicolumn{4}{|l|}{ Cardiopulmonary investigations } \\
\hline Vital capacity $\%$ of predicted, mean (SD) ${ }^{*}$ & $96(82-109)$ & $94(21)$ & $99(24)$ \\
\hline DLCO $\%$ of predicted, mean (SD) ${ }^{*}$ & $62(50-75)$ & $62(18)$ & $67(13)$ \\
\hline UIP according HRCT, N (\%) & $70(31)$ & $57(30)$ & $13(37)$ \\
\hline NSIP according HRCT, N (\%) & $69(31)$ & $62(33)$ & $7(21)$ \\
\hline SPAP $>35 \mathrm{~mm} \mathrm{Hg}, \mathrm{N}(\%)$ & $43(19)$ & $40(21)$ & $3(9)$ \\
\hline LVEF, mean (SD) & $58(7.1)$ & $61(8)$ & $60(7)$ \\
\hline Pericardial fluid, N (\%) & $10(4)$ & $9(5)$ & $1(3)$ \\
\hline Arrhythmia, N (\%) & $55(24)$ & $50(27)$ & $5(15)$ \\
\hline Conduction defect, N (\%) & $21(9)$ & $20(11)$ & $1(3)$ \\
\hline 6-min walk distance, meters, mean (SD) & $498(136)$ & $490(142)$ & $540(93)$ \\
\hline CPET; maximum VO2\% of predicted, mean (SD) & $91(12)$ & $87(25)$ & $96(24)$ \\
\hline \multicolumn{4}{|l|}{ Immunosuppressive therapy } \\
\hline Current & $77(34)$ & $68(36)$ & $9(26)$ \\
\hline Previous & $88(39)$ & $72(38)$ & $16(46)$ \\
\hline SHAQ (0-3), median (IQR)† & $0.6(0.3-1.1)$ & $0.8(0.3-1.1)$ & $0.3(0-0.6)$ \\
\hline \multicolumn{4}{|l|}{ SF-36, median (IQR) $\ddagger$} \\
\hline Physical Component Summary Scale & $40.1(32-49.5)$ & $38(30.1-47.9)$ & $48.3(42.3-53.8)$ \\
\hline Mental Component Summary Scale & $51.5(40-56.1)$ & $51.3(39-56.1)$ & $54.4(40.1-56.5)$ \\
\hline \multicolumn{4}{|c|}{$\begin{array}{l}\text { * }<5 \% \text { missing. } \\
\dagger<10 \% \text { missing. } \\
\ddagger<15 \% \text { missing. } \\
\text { ANA, antinuclear antibody; DcSSc, diffuse cutaneous SSc; DLCO, diffusing capacity for carbon monoxide; MRSS, modified Rodnan Skin } \\
\text { Score; NSIP, non-specific interstitial pneumonia; SF-36, Short-Form-36; SHAQ, Scleroderma Health Assessment Questionnaire; SPAP, } \\
\text { systolic pulmonary arterial pressure; UIP, usual interstitial pneumonia; VO2 max, maximum volume oxygen. }\end{array}$} \\
\hline
\end{tabular}

One hundred and nineteen (53\%) patients visited an occupational therapist, and $80(35 \%)$ patients visited a social worker.
Medical interventions resulting from the care pathway

The frequency of specific interventions resulting from the care pathway is shown in figure 1 . In total, one or 
Figure 1 Number of patients with frequencies of any intervention as a result from the care pathway (see enclosed online supplementary file 1).

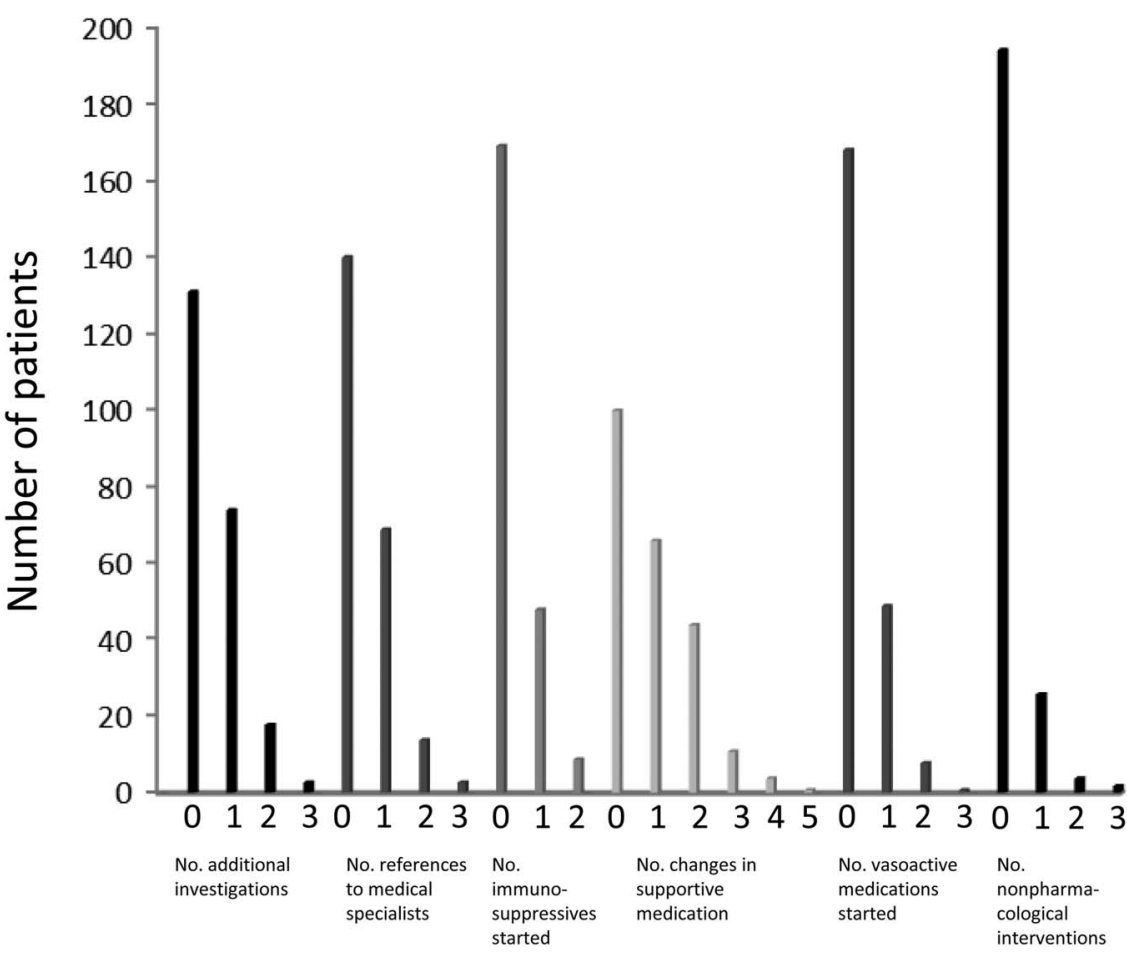

more additional diagnostic or therapeutic interventions were initiated in $191(85 \%)$ of the patients. In 95 patients $(42 \%)$, additional diagnostic tests were advocated. Among the patients with elevated SPAP (range 36-80 mm Hg; n=29: >35 mm Hg- $\leq 40$ mm Hg; n=10>40 mm Hg- $\leq 45 \mathrm{~mm} \mathrm{Hg} ; \mathrm{n}=17>45 \mathrm{~mm} \mathrm{Hg}$ ), six underwent RHC, which confirmed the diagnosis of PAH in four patients, resulting in initiation of bosentan. Two patients were classified as having pulmonary hypertension based on SPAP $\geq 50 \mathrm{~mm} \mathrm{Hg}$ by echocardiography alone $(n=1$ : SPAP $61 \mathrm{~mm} \mathrm{Hg}, \mathrm{n}=1$ SPAP $54 \mathrm{~mm} \mathrm{Hg}$ ) and started with bosentan without confirmation of actual pulmonary pressures by RHC, as the general condition of the patient did not allow such a procedure. The remaining patients with elevated SPAP were considered as either borderline pulmonary hypertension, and/or pulmonary hypertension due to left heart disease, or chronic lung disease. As no direct therapeutic intervention was expected following RHC, the multidisciplinary $\mathrm{PAH}$ group advised to repeat echocardiography within 612 months in these cases.

Stringent pulmonary follow-up of ILD was advised by additional PFT in 33 patients, and by additional HRCT thorax in 17 patients.

Additional examinations to obtain more detailed information about specific organ involvement was considered mandatory only in a minority of patients: colonoscopy $(n=7)$ and DXA scan $(n=6)$ were the investigations most frequently advised.

Eighty-eight patients $(39 \%)$ were referred to medical specialists. Patients were most frequently referred to a cardiologist $(n=17)$, a gastroenterologist $(n=16)$, a pulmonologist $(n=14)$ or a dermatologist $(n=14)$.

\section{Change of treatment}

At presentation, 77 patients $(34 \%)$ were using immunosuppressive medication. Based on the findings during the care pathway, new immunosuppressive treatment was started in 49 patients $(22 \%)$. Newly prescribed treatment included autologous HSCT, cyclophosphamide, mycophenolate mofetil (MMF), corticosteroids, methotrexate (MTX), AZA, HCQ, rituximab and rituximab/placebo. Two patients started with a combination of drugs: the first with cyclophosphamide and prednisone, and second started with MTX and was included in the RITIS trial. In one patient, treatment with cyclophosphamide was started, and it was advised to switch to MMF maintenance therapy after 6 monthly pulses (female, 27 years of age, wish to conceive in the future). Initiation of immunosuppressive therapy was advocated in presence of: ILD $(n=24)$, high or progressive skin score $(n=18)$, very early SSc ( $\leq 6$ months); puffy fingers $(n=3)$, arthritis $(n=5)$, renal involvement $(n=2)$, myositis $(n=1)$ and symptomatic pericardial effusion $(n=1)$. In none of the patients with previous HSCT, new immunosuppressive medication was started.

Advices on supportive medication most frequently consisted of changes (prescription, increase, decrease) in calcium channel blockers $(n=35)$ and ACE inhibitors $(n=33)$, or prescription of eye drops $(n=33)$. In 58 patients $(26 \%)$, medication for peripheral vascular complications was started, most often calcium channel blockers $(n=32)$. In $n=9$ patients, a combination of different vasoactive drugs and/or antibiotics was prescribed. Fifteen patients started with bosentan, 9 patients started with intravenous iloprost, and in $n=12$ patients antibiotic treatment for superimposed bacterial infections was prescribed. 
Table 2 Univariable and multivariable logistic regression analysis for patients with and without any medical intervention

\begin{tabular}{|c|c|c|c|c|}
\hline & $\begin{array}{l}\text { Univariable OR } \\
(95 \% \mathrm{CI})\end{array}$ & p Value & $\begin{array}{l}\text { Multivariable OR } \\
(95 \% \mathrm{Cl})\end{array}$ & p Value \\
\hline \multicolumn{5}{|l|}{ Sociodemographics } \\
\hline Age, years, mean (SD) & $1.02(0.99$ to 1.04$)$ & 0.241 & $1.00(0.98$ to 1.03$)$ & 0.823 \\
\hline Female, $\mathrm{N}(\%)$ & $1.05(0.40$ to 2.72$)$ & 0.925 & $1.36(0.50$ to 3.70$)$ & 0.554 \\
\hline Disease duration, years, median (IQR) & $1.01(0.96-1.06)$ & 0.699 & & \\
\hline Onset of RP, years, median (IQR) & $1.01(0.98-1.04)$ & 0.623 & & \\
\hline Onset of non-RP, years, median (IQR) & $1.02(0.97-1.07)$ & 0.471 & & \\
\hline \multicolumn{5}{|l|}{ Disease characteristics, N (\%) } \\
\hline DcSSc & $1.05(0.48$ to 2.28$)$ & 0.904 & & \\
\hline DU & $2.59(0.96$ to 7.02$)$ & 0.061 & & \\
\hline Telangiectasia & 2.19 (1.05 to 4.54$)$ & 0.036 & $2.27(1.08$ to 4.80$)$ & 0.031 \\
\hline Synovitis & $2.04(0.46$ to 9.11$)$ & 0.351 & & \\
\hline Friction rubs & $0.54(0.10$ to 2.77$)$ & 0.456 & & \\
\hline Proximal muscular weakness & $2.50(0.32$ to 19.73$)$ & 0.385 & & \\
\hline Pulmonary crackles & $1.73(0.71$ to 4.18$)$ & 0.226 & & \\
\hline MRSS, median (IQR) & $1.08(0.99-1.18)$ & 0.081 & & \\
\hline Anti-Scl-70 antibody & $1.44(0.59$ to 3.56$)$ & 0.426 & & \\
\hline Anticentromere antibody & $0.65(0.30$ to 1.40$)$ & 0.271 & & \\
\hline \multicolumn{5}{|l|}{ Diagnostic investigations } \\
\hline ESR, mm, median (IQR) & $1.04(1.01-1.07)$ & 0.013 & $1.04(1.01-1.07)$ & 0.012 \\
\hline CRP, mg/dL, median (IQR) & $1.06(0.96-1.16)$ & 0.246 & & \\
\hline CPK, mg/dL, median (IQR) & $1.00(1.00-1.00)$ & 0.743 & & \\
\hline Vital capacity \% of predicted, mean (SD) & $0.99(0.97$ to 1.01$)$ & 0.266 & & \\
\hline NSIP according HRCT, N (\%) & $1.80(0.74$ to 4.37$)$ & 0.195 & & \\
\hline $\begin{array}{l}\text { CPET; maximum VO2\% of predicted, } \\
\text { mean (SD) }\end{array}$ & 0.99 (0.97 to 1.00$)$ & 0.061 & & \\
\hline
\end{tabular}

\section{Determinants of medical interventions}

In total, one or more medical interventions (defined as: (1) need for additional diagnostic follow-up, (2) referral to medical specialists, (3) referral to other healthcare professionals or (4) initiation of immunosuppressive treatment and change of supportive medications), were initiated in $191(85 \%)$ of patients as a result of the care pathway.

Multivariable logistic regression analyses showed that patients with medical interventions had a higher ESR, and more often had telangiectasias than patients without any medical intervention (table 2).

\section{Determinants of initiation of immunosuppressive therapy}

Univariate logistic regression analysis of disease characteristics, and results of diagnostic investigations showed that younger age, DcSSc, shorter duration of disease and (non-) RP, proximal muscular weakness, dyspnoea, anti-Scl-70, pulmonary crackles, NSIP, higher mRSS, ESR, CRP and CPK, and lower VC, wattage and $\mathrm{VO}_{2}$ max at CPET, were significantly associated with the start of new immunosuppressive therapy. Presence of telangiectasias, and ACA decreased the chance for start of new immunosuppressive therapy (table 3 ).

Multivariable analysis showed that next to NSIP according to HRCT, CPK, mRSS and age were associated with start of immunosuppressive treatment, with NSIP according to HRCT having the largest impact (table 3). Repeated multivariate testing including only variables resulting from history taking, physical examination and laboratory testing identified age (OR 0.96 ; $95 \%$ CI ( 0.93 to $0.99)$ ), skin score (mRSS; OR 1.12; 95\% CI (1.04 to 1.21)), and presence of ACA (OR 0.19; $95 \%$ CI 0.04 to 0.85$)$ ) as most significantly associated with initiation of immunosuppressive therapy. Higher age and presence of ACA decreased the change of start of immunosuppressives.

Non-medical interventions resulting from the care pathway Forty-three patients (19\%) were referred to other healthcare professionals, most frequently to a physical therapist in primary setting $(\mathrm{n}=26)$ for an exercise programme, and/or improvement of physical functioning, an occupational therapist $(n=7)$ for assistive devices and/or adjustments at home, or a dietician $(n=7)$.

Non-pharmacological treatment advices provided by the specialised nurse included therapeutic gloves $(9 \%$ silver; $n=16)$, stopping smoking $(n=13)$, and other lifestyle advices including dietary advices $(n=5)$.

\section{Patient satisfaction}

In total, 96 patients (42\%) returned the questionnaire concerning different aspects of the care pathway. 
Table 3 Univariable and multivariable logistic regression analysis for patients who started and did not start with immunosuppressive therapy as a result from the care pathway

\begin{tabular}{|c|c|c|c|c|c|c|}
\hline & $\begin{array}{l}\text { Start } \\
\mathrm{N}=49\end{array}$ & $\begin{array}{l}\text { Not started } \\
\mathrm{N}=177\end{array}$ & $\begin{array}{l}\text { Univariable OR } \\
(95 \% \mathrm{Cl})\end{array}$ & $\mathrm{p}$ Value & $\begin{array}{l}\text { Multivariable OR } \\
(95 \% \mathrm{Cl})\end{array}$ & p Value \\
\hline \multicolumn{7}{|l|}{ Sociodemographics } \\
\hline Age, years, mean (SD) & $48(15)$ & $56(14)$ & 0.96 (0.94 to 0.98$)$ & $<0.001$ & 0.94 (0.91 to 0.98$)$ & 0.005 \\
\hline Female, $\mathrm{N}(\%)$ & $38(78)$ & $140(86)$ & 1.76 (0.79 to 3.93$)$ & 0.167 & & \\
\hline $\begin{array}{l}\text { Disease duration, years, median } \\
\text { (IQR) }\end{array}$ & $1(0-4)$ & $4(1-12)$ & $0.93(0.88$ to 0.98$)$ & 0.012 & & \\
\hline Onset of RP, years, median (IQR) & $6(2-16)$ & $13(6-21)$ & 0.96 (0.93 to 0.99$)$ & 0.020 & & \\
\hline $\begin{array}{l}\text { Onset of non-RP, years, median } \\
\text { (IQR) }\end{array}$ & $3(1-5)$ & $7(3-13)$ & 0.94 (0.89 to 0.99$)$ & 0.016 & & \\
\hline \multicolumn{7}{|l|}{ Disease characteristics, $\mathrm{N}(\%)$} \\
\hline DcSSc & $26(53)$ & $33(56)$ & 4.45 (2.26 to 8.78$)$ & $<0.001$ & $1.33(0.35$ to 5.05$)$ & 0.676 \\
\hline Digital ulcers & $11(22)$ & $49(30)$ & $0.66(0.31$ to 1.40$)$ & 0.281 & & \\
\hline Telangiectasia & $20(41)$ & $107(66)$ & 0.35 (0.18 to 0.68$)$ & 0.002 & $0.46(0.16$ to 1.30$)$ & 0.155 \\
\hline Synovitis & $8(16)$ & $15(9)$ & $1.93(0.76$ to 4.86$)$ & 0.165 & & \\
\hline Friction rubs & $4(8)$ & 4 (3) & $3.53(0.85$ to 14.69$)$ & 0.083 & & \\
\hline Proximal muscular weakness & $8(16)$ & $6(4)$ & $5.07(1.67$ to 15.44$)$ & 0.004 & $5.06(0.93$ to 27.68$)$ & 0.061 \\
\hline Pulmonary crackles & $21(44)$ & 36 (22) & $2.72(1.38$ to 5.37$)$ & 0.004 & $2.44(0.74$ to 8.02$)$ & 0.142 \\
\hline mRSS, median (IQR) & $5(2-20)$ & $2(0-5)$ & $1.13(1.07$ to 1.18$)$ & $<0.001$ & $1.09(1.00$ to 1.01$)$ & 0.042 \\
\hline Anti-Scl-70 antibody & $18(37)$ & $28(17)$ & 4.07 (1.88 to 8.82$)$ & $<0.001$ & & \\
\hline Anticentromere antibody & $4(8)$ & $72(44)$ & $0.11(0.04$ to 0.33$)$ & $<0.001$ & & \\
\hline \multicolumn{7}{|l|}{ Diagnostic investigations } \\
\hline ESR, mm, median (IQR) & $19(11-41)$ & $14(6-28)$ & $1.02(1.00$ to 1.03$)$ & 0.033 & & \\
\hline CRP, mg/dL, median (IQR) & $3(3-7)$ & $3(3-4)$ & 1.04 (1.01 to 1.07$)$ & 0.020 & & \\
\hline CPK, mg/dL, median (IQR) & $95(54-174)$ & $85(61-114)$ & $1.01(1.00$ to 1.01$)$ & 0.009 & $1.01(1.000$ to 1.01$)$ & 0.039 \\
\hline $\begin{array}{l}\text { Vital capacity \% of predicted, } \\
\text { mean (SD) }\end{array}$ & $86(19)$ & $98.6(21)$ & 0.97 (0.95 to 0.99$)$ & $<0.001$ & & \\
\hline NSIP according HRCT, N (\%) & $27(55)$ & $36(22)$ & $4.13(2.10$ to 8.10$)$ & $<0.001$ & $\begin{array}{l}4.89(1.561 \text { to } \\
15.30)\end{array}$ & 0.006 \\
\hline $\begin{array}{l}\mathrm{CPET} \text {; maximum VO2\% of } \\
\text { predicted, mean (SD) }\end{array}$ & $79(20)$ & $91(26)$ & 0.98 (0.97 to 0.99$)$ & 0.006 & & \\
\hline
\end{tabular}

Between the patients that completed and returned the questionnaire, and the patients who did not return the questionnaire, there was no difference in age, sex, disease duration, frequency of start of supportive or immunosuppressive medication, and frequency of referral to medical specialists. Patients who did not return the questionnaire more often had DcSSc $(n=52(40 \%)$, vs $\mathrm{n}=21(22 \%) ; \mathrm{p}=0.004)$, and were more often referred to allied health professionals $(\mathrm{n}=35(27 \%)$, vs $8(8 \%)$; $\mathrm{p}<0.001)$. In general, patients were very satisfied with the care programme, resulting in a mean grade of 9 on a scale of 0 to $10(0=$ lowest, $10=$ highest grade $)$. More specifically, $88 \%$ of patients $(\mathrm{n}=84)$ stated that the care programme was in line with their healthcare needs, $91 \%$ of patients $(n=87)$ judged the time schedule as being sufficient, and the percentage of patients being satisfied/ very satisfied with the different healthcare providers was high (rheumatologist $n=94 ; 96 \%$, specialised nurse $n=93$; $96 \%$, physical therapist $\mathrm{n}=87 ; 89 \%$, occupational therapist $\mathrm{n}=51,96 \%$, social worker $\mathrm{n}=29 ; 82 \%$ ) (numbers/ percentages differ, as not all patients visited the occupational therapist and the social worker).

\section{DISCUSSION}

The present study describes the medical management of 226 patients with SSc referred to a 2-day comprehensive diagnostic care pathway. In $191(85 \%)$ patients, the care pathway resulted in specific advices on additional diagnostic follow-up, referral or change of treatment. Of patient characteristics only telangiectasias and ESR were associated with any medical intervention. Commonly available patient characteristics associated with change of immunosuppressives included lower age, absence of ACA and higher mRSS. These data indicate that a standardised comprehensive care pathway is of benefit for the far majority of patients with SSc, regardless of SSc subtype and disease duration. Systematic organ screening has been shown to contribute to survival of patients with SSc over the past decades, ${ }^{3}$ mainly in patients with DcSSc. This observation, in combination with preference of the patients with SSc to combine different diagnostic procedures and visits to medical specialists in a day-care setting, ${ }^{14}$ and high health needs despite frequent consultations, ${ }^{28}$ led us to implement the described day-care programme. In general, adherence to the care pathway 
was high and patient satisfaction rates were very high. This latter finding probably reflects the successful match between patients' needs and care provision, as the care pathway was developed based on the questionnaire on patients' needs and preferences. ${ }^{14}$ Whether implementation of the described care pathway will contribute to diminish the number of visits to the hospital in the long run, remains to be determined. Most likely, benefits in terms of medical interventions will be numerically different after following annual visits. Future evaluations will have to demonstrate in which subgroups of patients repeated extensive annual screening is most useful and whether standardised and multidisciplinary care as applied in the care pathway will contribute to improved outcome.

As one or more medical interventions took place in 191 of 226 patients, the care pathway did not result in any specific medical intervention in 1 of $6-7$ patients. To evaluate in which patients referral to the care pathway was most useful, multivariable regression analyses were performed. Presence of telangiectacias was associated with need for any medical intervention, while it was protective for starting immunosuppressive treatment. It is known that presence of telangiectacias is associated with vascular complications including digital ulcers and pulmonary hypertension. ${ }^{29}$ Indeed, subgroup comparisons within our own cohort between patients with telangiectasias and patients without telangiectasias showed that patients with telangiectasias had more often calcinosis, more often had a change in vasoactive medication, and they were discussed more frequently in the $\mathrm{PAH}$ meeting. Patients without telangiectasias needed more stringent follow-up on pulmonary fibrosis reflected by need for additional PFTs (see online supplementary table S1). This observation is thus in line with previous studies showing the association between vascular complications and telangiectasias in SSc. ${ }^{29}$ Consequently, the number and type of interventions resulting from the care pathway is most likely different for the different subgroups of SSc, as reflected by the differential association of telangiectasias with the different interventions.

Our study confirms previous findings about clinically important investigations in evaluation of severity and activity of SSc. ${ }^{30}$ Multivariable analysis showed that age, mRSS, proximal muscle weakness, CPK and NSIP, according to HRCT-thorax were significantly associated with the start of new immunosuppressive therapy. These findings are explained by the fact that skin involvement and pulmonary involvement were the most frequent indications for immunosuppressive treatment, and thus, reflect a certain circularity. In addition, muscle involvement was identified as a relevant parameter, reflected by both CPK and proximal muscle weakness. SSc overlap with myositis is found in approximately $15 \%$ of the patients with SSc, ${ }^{31}$ and is associated with more extensive disease. ${ }^{32}$ Although these parameters seem to be rather non-specific, two important recent studies also identified CPK level and myopathy as important predictors for worse outcome. ${ }^{33} 34$ As no circularity can be involved specifically for these two parameters, we do believe that our data confirm previous data on CPK and myopathy being important predictors for worse prognosis, and thus, should probably be taken into account in therapeutic decision-making.

By contrast with previous studies, no attributive value for start of immunosuppressive therapy of type of SSc, PFTs and cardiac parameters was found. ${ }^{8}$ Several factors can account for a lack of association between cardiac parameters and of immunosuppressive treatment. First, only in case of active myocarditis, immunosuppressive treatment is advocated..$^{35}$ In case of diastolic dysfunction, or significant arrhytmias, primary interventions consist of ACE inhibitors, antiarrhythmics and placement of implantable cardioverter defibrillator where appropriate. Finally, the included diagnostic procedures might not have revealed subclinical myocardial involvement, as plain echocardiography is rather insensitive for this goal, ${ }^{36}$ and the majority of patients already received treatment with calcium channel blockers and/or ACE inhibitors for different indications. Another interesting finding was that, apart from consultations with a nurse and physical therapist, more than half the patients took the opportunity to visit an occupational therapist and/ or social worker. This observation shows that many patients encounter problems which can clearly not be solved by medical treatment alone. The relatively high proportion of patients in the present study expressing a need to see additional healthcare providers again underscores that more research into the most appropriate non-pharmacological management strategies in this patient group is warranted. ${ }^{37}$

This study has a number of limitations which should be taken into account when interpreting the results. First, selection bias cannot be excluded, as patients referred to the care programme in a tertiary care setting might not represent the total population of patients with SSc. However, comparison with other cohorts of patients with SSc evaluated by tertiary care centres, including the EUSTAR centres, showed that social and demographic characteristics, disease severity and functional status of our cohort are quite similar. ${ }^{8}$ At admission, $34 \%$ of the patients were treated with immunosuppressive therapy, which is also comparable to reports from the EUSTAR cohort. ${ }^{39}$ Second, start of immunosuppressive therapy was based on consensus between experts during a multidisciplinary discussion, in the absence of a gold standard precisely guiding treatment decisions in SSc. ${ }^{15}$ Whether treatment decisions in the current cohort were adequate remains to be determined. Ideally, one should dispose of a control group receiving standard care to compare number of medical interventions, progression-free survival and quality of life. Finally, the current study describes results of the care path way at short term. Long-term follow-up is needed to evaluate the impact on patients' health status and healthcare usage. These results could guide further improvements of the contents and organisation of care. 
In conclusion, our data demonstrate that a comprehensive diagnostic and multidisciplinary care pathway for patients with SSc in a day-care setting, combining as such annual screening on organ involvement as indicated according to (inter) national guidelines with multidisciplinary team care, is feasible. In general, adherence to the care pathway was very high, and resulted in medical interventions in the majority of patients, independent of SSc subtype and disease duration. Future evaluations are needed to evaluate impact on disease outcome.

\section{Author affiliations}

${ }^{1}$ Department of Rheumatology, Leiden University Medical Center, Leiden, The Netherlands

${ }^{2}$ Haga Hospital, The Hague, The Netherlands

${ }^{3}$ Department of Cardiology, Leiden University Medical Center, Leiden, The Netherlands

${ }^{4}$ Department of Radiology, Leiden University Medical Center, Leiden, The Netherlands

${ }^{5}$ Department of Medical Statistics, Leiden University Medical Center, Leiden, The Netherlands

${ }^{6}$ Department of Pulmonology, Leiden University Medical Center, Leiden, The Netherlands

${ }^{7}$ Department of Orthopaedics, Leiden University Medical Center, Leiden, The Netherlands

Contributors JM collected the data, was involved in statistical analysis, writing and critically reviewing the manuscript and approved the final version. AAS collected the data, was involved in critically reviewing the manuscript and approved the final version. NAM collected the data, was involved in critically reviewing the manuscript, and approved the final version. LJMK was involved in CT data acquisition, critically reviewing the manuscript, and approved the final version. TS was involved in statistical analysis, and approved the final version. MKN collected the data, was involved in critically reviewing the manuscript, and approved the final version. TWJH reviewed the data statistics, was involved in critically reviewing the manuscript, and approved the final version. TPMVV was involved in the design of the study, was involved in writing and critically reviewing the manuscript, and approved final version. JKdV-B reviewed the data statistics, was involved in statistical analysis, writing and critically reviewing the manuscript, and approved the final version.

Competing interests JM was supported by an unrestricted educational grant of Actelion Pharmaceuticals Nederland BV (Woerden, The Netherlands).

Patient consent Obtained.

Ethics approval Institutional Review Board of the LUMC.

Provenance and peer review Not commissioned; externally peer reviewed.

Data sharing statement No additional data are available.

Open Access This is an Open Access article distributed in accordance with the Creative Commons Attribution Non Commercial (CC BY-NC 4.0) license, which permits others to distribute, remix, adapt, build upon this work noncommercially, and license their derivative works on different terms, provided the original work is properly cited and the use is non-commercial. See: http:// creativecommons.org/licenses/by-nc/4.0/

\section{REFERENCES}

1. Elhai M, Meune C, Avouac J, et al. Trends in mortality in patients with systemic sclerosis over 40 years: a systematic review and meta-analysis of cohort studies. Rheumatology (Oxford) 2012;51:1017-26.

2. Steen VD, Medsger TA. Severe organ involvement in systemic sclerosis with diffuse scleroderma. Arthritis Rheum 2000;43:2437-44.

3. Nihtyanova SI, Tang EC, Coghlan JG, et al. Improved survival in systemic sclerosis is associated with better ascertainment of internal organ disease: a retrospective cohort study. QJM 2010;103:109-15.

4. Nihtyanova SI, Schreiber BE, Ong VH, et al. Prediction of pulmonary complications and long-term survival in systemic sclerosis. Arthritis Rheum 2014;66:1625-35.

5. Coghlan JG, Denton CP, Grunig E, et al. Evidence-based detection of pulmonary arterial hypertension in systemic sclerosis: the DETECT study. Ann Rheum Dis 2014;73:1340-9.

6. Goh NS, Desai SR, Veeraraghavan S, et al. Interstitial lung disease in systemic sclerosis: a simple staging system. Am J Respir Crit Care Med 2008;177:1248-54.

7. Valentini $\mathrm{G}$. The assessment of the patient with systemic sclerosis. Autoimmun Rev 2003;2:370-6.

8. Walker UA, Tyndall A, Czirjak L, et al. Clinical risk assessment of organ manifestations in systemic sclerosis: a report from the EULAR Scleroderma Trials and Research group database. Ann Rheum Dis 2007;66:754-63.

9. Khanna D, Denton CP. Evidence-based management of rapidly progressing systemic sclerosis. Best Pract Res Clin Rheumatol 2010;24:387-400.

10. Mierau R, Moinzadeh P, Riemekasten G, et al. Frequency of disease-associated and other nuclear autoantibodies in patients of the German Network for Systemic Scleroderma: correlation with characteristic clinical features. Arthritis Res Ther 2011;13:R172.

11. Moinzadeh $\mathrm{P}$, Nihtyanova SI, Howell K, et al. Impact of hallmark autoantibody reactivity on early diagnosis in scleroderma. Clin Rev Allergy Immunol 2012;43:249-55.

12. Willems LM, Vriezekolk JE, Schouffoer AA, et al. Effectiveness of nonpharmacologic interventions in systemic sclerosis: a systematic review. Arthritis Care Res (Hoboken) 2015;67:1426-39.

13. Avouac J, Kowal-Bielecka O, Landewe R, et al. European League Against Rheumatism (EULAR) Scleroderma Trial and Research group (EUSTAR) recommendations for the treatment of systemic sclerosis: methods of elaboration and results of systematic literature research. Ann Rheum Dis 2009;68:629-34.

14. Schouffoer AA, Zirkzee EJ, Henquet SM, et al. Needs and preferences regarding health care delivery as perceived by patients with systemic sclerosis. Clin Rheumatol 2011;30:815-24.

15. Kowal-Bielecka O, Landewe R, Avouac J, et al. EULAR recommendations for the treatment of systemic sclerosis: a report from the EULAR Scleroderma Trials and Research group (EUSTAR). Ann Rheum Dis 2009;68:620-8.

16. [No authors listed]. Preliminary criteria for the classification of systemic sclerosis (scleroderma). Subcommittee for scleroderma criteria of the American Rheumatism Association Diagnostic and Therapeutic Criteria Committee. Arthritis Rheum 1980;23:581-90.

17. Leroy EC, Medsger TA Jr. Criteria for the classification of early systemic sclerosis. J Rheumatol 2001;28:1573-6.

18. Medsger TA Jr. Natural history of systemic sclerosis and the assessment of disease activity, severity, functional status, and psychologic well-being. Rheum Dis Clin North Am 2003;29:255-73, vi.

19. Cutolo M, Sulli A, Pizzorni C, et al. Nailfold videocapillaroscopy assessment of microvascular damage in systemic sclerosis. J Rheumatol 2000;27:155-60.

20. Clements PJ, Wong WK, Hurwitz EL, et al. The Disability Index of the Health Assessment Questionnaire is a predictor and correlate of outcome in the high-dose versus low-dose penicillamine in systemic sclerosis trial. Arthritis Rheum 2001;44:653-61.

21. ATS Committee on Proficiency Standards for Clinical Pulmonary Function Laboratories. ATS statement: guidelines for the six-minute walk test. Am J Respir Crit Care Med 2002;166:111-17.

22. Ware JJ, Snow K, Kosinki M. SF-36 Health survey manual and interpretation guide. Boston, MA: The Health Institute, New England Medical Center, 1994.

23. Newnham EA, Harwood KE, Page AC. Evaluating the clinical significance of responses by psychiatric inpatients to the mental health subscales of the SF-36. J Affect Disord 2007;98:91-7.

24. American Thoracic Society/European Respiratory Society International Multidisciplinary Consensus Classification of the Idiopathic Interstitial Pneumonias. This joint statement of the American Thoracic Society (ATS), and the European Respiratory Society (ERS) was adopted by the ATS board of directors, June 2001 and by the ERS Executive Committee, June 2001. Am J Respir Crit Care Med 2002;165:277-304.

25. Rudski LG, Lai WW, Afilalo J, et al. Guidelines for the echocardiographic assessment of the right heart in adults: a report from the American Society of Echocardiography endorsed by the European Association of Echocardiography, a registered branch of the European Society of Cardiology, and the Canadian Society of Echocardiography. J Am Soc Echocardiogr 2010;23:685-713. 
26. Vacca A, Meune C, Gordon J, et al. Cardiac arrhythmias and conduction defects in systemic sclerosis. Rheumatology 2014;53:1172-7.

27. van Laar JM, Farge D, Sont JK, et al. Autologous hematopoietic stem cell transplantation vs intravenous pulse cyclophosphamide in diffuse cutaneous systemic sclerosis: a randomized clinical trial. JAMA 2014;311:2490-8.

28. Meijs J, Zirkzee EJ, Schouffoer AA, et al. Health-care utilization in Dutch systemic sclerosis patients. Clin Rheumatol 2014;33:825-32.

29. Zhang SZ, Xu D, Li MT, et al. Telangiectasia as a potential clinical marker of microvascular lesions in systemic sclerosis patients from EUSTAR data. Clin Exp Rheum 2015;33(4 Suppl 91):S106-10.

30. Khanna D, Kowal-Bielecka O, Khanna PP, et al. Quality indicator set for systemic sclerosis. Clin Exp Rheumatol 2011;29(2 Suppl 65): S33-9.

31. Muangchan C, Baron M, Pope J. The $15 \%$ rule in scleroderma: the frequency of severe organ complications in systemic sclerosis. A systematic review. J Rheumatol 2013;40:1545-56.

32. Schade L, Paiva ES, Muller CS. Skeletal and cardiac muscles involvement in systemic sclerosis. Rev Bras Reumatol 2011;51:309-10, 313.

33. Maurer B, Graf N, Michel BA, et al. Prediction of worsening of skin fibrosis in patients with diffuse cutaneous systemic sclerosis using the EUSTAR database. Ann Rheum Dis 2015;74:1124-31.
34. Jung M, Bonner A, Hudson M, et al. Myopathy is a poor prognostic feature in systemic sclerosis: results from the Canadian Scleroderma Research Group (CSRG) cohort. Scand J Rheum 2014;43:217-20.

35. Pieroni M, De Santis M, Zizzo G, et al. Recognizing and treating myocarditis in recent-onset systemic sclerosis heart disease: potential utility of immunosuppressive therapy in cardiac damage progression. Semin Arthritis Rheum 2014; 43:526-35.

36. Sandmeier B, Jäger VK, Nagy G, et al. Autopsy versus clinical findings in patients with systemic sclerosis in a case series from patients of the EUSTAR database. Clin Exp Rheumatol 2015;33(4 Suppl 91):S75-9.

37. Willems LM, Kwakkenbos L, Leite CC, et al. Frequency and impact of disease symptoms experienced by patients with systemic sclerosis from five European countries. Clin Exp Rheumatol 2014;32 (Suppl 86):88-93.

38. Vanthuyne M, Smith V, De Langhte E, et al. The Belgian Systemic Sclerosis Cohort: correlations between disease severity scores cutaneous subsets, and autoantibody profile. J Rheumatol 2012;39:2127-33.

39. Avouac J, Walker UA, Hachulla E, et al. Joint and tendon involvement predict disease progression in systemic sclerosis: a EUSTAR prospective study. Ann Rheum Dis 2016;75:103-9. 